\title{
The Effect of Uncertainty in Whole Building Simulation Models for Purposes of Generating Control Strategies
}

\author{
Amar Kumar SEEAM ${ }^{\mathrm{a}, 1}$, David LAURENSON $^{\mathrm{b}}$ and Asif USMANI ${ }^{\mathrm{c}}$ \\ ${ }^{a}$ Middlesex University Mauritius, Mauritius \\ ${ }^{\mathrm{b}}$ The University of Edinburgh, Scotland, United Kingdom \\ ${ }^{\mathrm{c}}$ Hong Kong Polytechnic University, Hong Kong
}

\begin{abstract}
Buildings consume a significant amount of energy worldwide in maintaining comfort for occupants. Building energy management systems (BEMS) are employed to ensure that the energy consumed is used efficiently. However these systems often do not adequately perform in minimising energy use. This is due to a number of reasons, including poor configuration or a lack of information such as being able to anticipate changes in weather conditions. We are now at the stage that building behaviour can be simulated, whereby simulation tools can be used to predict building conditions, and therefore enable buildings to use energy more efficiently, when integrated with BEMS. What is required though, is an accurate model of the building which can effectively represent the building processes, for building simulation. Building information modelling (BIM) is a relatively new method of representing building models, however there still remains the issue of data translation between a BIM and simulation model, which requires calibration with a measured set of data. If there a lack of information or a poor translation, a level of uncertaintly is introduced which can affect the simulation's ability to accurate predict control strategies for BEMS. This paper explores effects of uncertainty, by making assumptions on a building model due to a lack of information. It will be shown that building model calibration as a method of addressing uncertainty is no substitute for a well defined model.
\end{abstract}

Keywords. building model, calibration, uncertainty, ESP-r

\section{Introduction}

Intelligent Buildings have recently become a focus of attention for sustainable living, with their promises of optimal efficiency and comfort, using zero energy to solve these issues. The literature describes these buildings as being self-sufficient, even self-organising and sentient and in some cases can be true prosumers, a promising theory supported by the development of an emerging smart grid infrastructure, where buildings produce more energy than they consume. Variables which can be adjusted in a building to maintain adequate comfort in an energy efficient way, often relate to the thermal, lighting and air

\footnotetext{
${ }^{1}$ Corresponding Author: Middlesex University Mauritius, Coastal Road, 90203, Uniciti, Mauritius; E-mail: a.seeam@mdx.ac.mu.
} 
quality characteristics of the building. These variables are required to be constantly monitored so that they can be manipulated to make the building comfortable to work or live in, and there are a wide range of computer controlled systems that are used to achieve this. We are now at the point where building models can be coupled with BEMS to help aid in prediction of control outcomes. However, before a building model can be used to generate predictive control strategies or perform energy auditing when coupled to a building management system, it needs to be accurately modeled and calibrated with the data gathered from previous Buiding Information Model (BIM) data, and monitored data from sensors instrumented in the building that is to be modeled. Notably current building energy management systems are not as dynamic as they could be, and in even in real world use, are often neither correctly used, nor optimised for energy efficiency. Changes in the environment (both internal and external) can affect the operation, and over time settings drift to inefficient boundaries, leading to situations that make occupants uncomfortable and a waste of energy. Simulation assisted control (SAC) utilises pre-existing (i.e. white-box) building models, which fully represent the building in terms of their geometry, operations and constructions and can have their energy performance and thermodynamic and airflow behaviour predicted using building energy performance simulation (BEPS) tools, such as ESP-r [1], used in this study. These predictions can then be used to formulate energy efficient control strategies, such as optimum heat startup, taking full consideration of all potential physical processes in a building, and are not constrained by the range of experience learned by Model Predictive Control (MPC) techniques $[2,3,4]$ from training data, which would otherwise only consider a subset of the building's true energy performance. Building information modelling (BIM) is an emerging discipline which can potentially aid in providing the required information needed to create BEPS models. Essentially BIM is an extension of 3D CAD, with supplementary building specific information, though they require further translation in order to represent the additional nuances required in BEPS models, such as the processing of architectural geometry into thermal boundaries and zones. A lack of information or approximation of the geometry or constructions may require calibration to a set of data to address uncertainty (therefore equivalent to a grey box model, which assumes some level of knowledge). Converting BIMs to be used in BEPS tools currently requires a degree of human intervention during the translation (i.e. semi-automated), due to the intricacy required in assigning BEPS specific details. If the process is automated without human intervention, an approximate model may be produced, which may require further calibration with measured data to tune the model and 'fill the gaps' with BEPS specific details not contained in the original BIM. These approximations may create various levels of uncertainty that lead to a model that is further divergent from reality, but may appear plausible in some cases, if not fully investigated. Furthermore, there is the issue of quality of data in terms of information provided to create the building model. That is, calibration can be used as a means to reduce uncertainty, when faced with a lack of information, which can rectify the model according to the data provided, but may be limited in scope and application since there may be a dependency on the measured data. In other words, calibration may not be the best approach when creating models, particularly if detailed data can be attained. The next section provides some background to the problem; Section 3 will contextualise the methodology used, Section 4 describe the results of introducing uncertainty and the final section will conclude the paper. The main contribution of this paper is to demonstrate that calibration is no substitute for a highly detailed model, with accurate construction 
data. That is to say, it will be demonstrated that calibration as a method of addressing uncertainty is no substitute for a well defined model, and calibration eventually will lead to skewed results, rendering a BEMS ineffective for the purposes of accurate prediction.

\section{Background}

Before a building model can be used to generate predictive control strategies or perform energy auditing when coupled to a building management system, it needs to be accurately modeled and calibrated with the data gathered from previous BIM data, and monitored data from BEMS sensors instrumented in the building that is to be modeled.The procedures to calibrate a building model using measured BEMS data will be discussed. Building simulation calibration is important to yield an accurate usable model. The accuracy of the model can be determined by comparing simulated and measured data using several metrics. Calibration is not a trivial problem, as energy models are complex with many interactions [5]. In the area of building simulation research, this is a deep and challenging problem, largely dependent on the quality of measured data available [6]. Most calibration methods reported in the literature pertain to commercial buildings which typically have higher-stake retrofit measure considerations than residential buildings [7]. That is the potential that could yield significant improvements to retrofit more efficient HVAC is greater for commercial applications.

\section{Calibration Methodology}

Calibration involves modifying model input parameters, in a sytematic way, until the model has passed a threshold to be deemed calibrated. It has been said that calibration, relies on user knowledge, past experience, statistical expertise, engineering judgement, and an abundance of trial and error [8].There are several approaches and methodologies which can be followed. The ASHRAE 1051-RP project [9] was an attempt to define a method to improve the process of calibrating whole building energy simulation models using monthly utility data. 1051-RP used the ASHRAE Guideline 14 [10] which states goodness of fit criteria (CV(RMSE)) to assist calibration, specified under section 5.2.11.3 (Modeling Uncertainty), and is typically the main methodology applied in similar studies. CV (RMSE) (Coefficient of Variation of the Root Mean Squared Error) measures the differences between simulated $(s)$ and measured $(m)$ values, at each timestep $i$, for a total number of timesteps, $n$. A lower value indicates less variance and hence higher quality model. CV(RMSE) aggregates time specific errors into a single dimensionless number.

Coefficient of variation of the root mean square error (CV(RMSE))

$$
\begin{aligned}
& R M S E=\sqrt{\frac{\sum_{i=1}^{n}\left(m_{i}-s_{i}\right)^{2}}{n}} \\
& C V(R M S E)=\frac{R M S E}{\bar{m}} .100
\end{aligned}
$$

$\mathrm{CV}(\mathrm{RMSE})$ of $15 \%$ is acceptable for calibration models and $30 \%$ for hourly models. Hourly data gives the most accurate results, though is the most difficult to capture; 
monthly data can also be acceptable depending on the application, but can mask inaccuracies that can appear at hourly or daily resolutions [11]. The coefficient of determination, $R^{2}$ has also seen use in work by Tahmasebi for optimisation, [12], to assess models' goodness-of-fit for simulation assisted control, based on temperature calibration. $R^{2}$ provides an indication of how well observed outcomes are replicated by the simulation model. The coefficient of determination ranges from 0 to 1 , with a value of 1 indicating that the model is a perfect fit. ASHRAE guideline 14 is widely used to help calibration and clearly defines statistic indexes as thresholds for calibrated model. The majority of literature in the area of building model calibration have thus adopted these conditions and statistical measures.

\section{Coefficient of determination $R^{2}$}

$$
R^{2}=\frac{\Sigma m_{i} s_{i}-\Sigma m_{i} \Sigma s_{i}}{\sqrt{\left(n \Sigma\left(m_{i}^{2}-\left(\Sigma m_{i}\right)^{2}\right)\left(n \Sigma s_{i}^{2}-\left(\Sigma s_{i}\right)^{2}\right)\right.}}
$$

\section{Results}

In this section, uncertainty will be introduced into an ESP-r building model (based on [13]) and an attempt at calibration will made using measured data. In this model, we have a whole domestic house, with scheduled heating systems in an Office space and Living Room. This will represent an analogy to a grey box model, which assumes some level of building knowledge. The effects of uncertainty will be analysed, by making assumptions on the building model due to a lack of information. It will be shown that uncertainty in a building model needs to be minimised by having as much information as possible, and that building model calibration as a method of addressing uncertainty is no substitute for a well defined model.

\subsection{Reasons for Uncertainty}

There could be number of reasons for uncertainty in a model.

1. A basic translation from a BIM. Geometry translation from BIM to BEPS models can lead to a loss of information.

2. Lack of source data about the building construction.

In either case, calibration is required to adjust the model accordingly, by comparing the output of the model with measured data. Calibration techniques and approaches will also be covered and discussed. There can be several sources of uncertainty in a model. The following sections will describe the types of uncertainty introduced in the model according to those identified by [14] in an "Analysis of uncertainty in building design evaluations and its implications".

\subsection{Specification Uncertainty}

Relates to a lack of information on the exact properties of the building, such as the building geometry. In the uncertain model the roof has been removed (Figure 1), approximating the geometry of the model. 


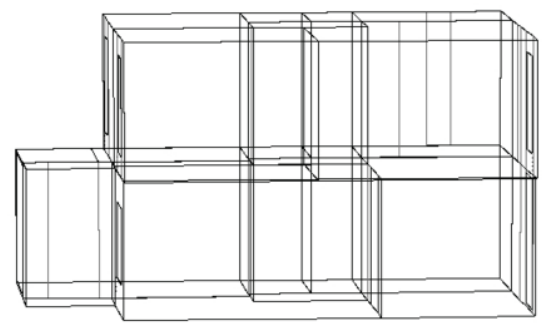

Figure 1. Geometric approximation - no roof.

\subsection{Parameter Uncertainty}

There can be degree of a uncertainty for each input parameter, for example, material properties.

\subsection{Modelling Uncertainty}

Arises from simplifications and assumptions that have been been introduced in the development of the model. Thus in the uncertain model there is no fluid flow model for air applied, though scheduled airflow is explored, further simplifying the building dynamics and physical processes.

\subsection{Model Calibration}

To reduce uncertainty, calibration techniques need to be applied. Calibration involves modifying model input parameters, in a systematic way, until the model has passed a threshold to be deemed "calibrated", according to criteria set out by the American Society of Heating, Refrigerating, and Air-Conditioning Engineers (ASHRAE) in ASHRAE Guideline 14 [10] which uses CV(RMSE) to assist calibration, specified under section 5.2.11.3 (Modelling Uncertainty). The ASHRAE guidelines are often used to benchmark building models in the majority of calibration and validation studies for building simulation. According to the criteria, a CV(RMSE) of $15 \%$ is acceptable for calibration models using monthly data and $30 \%$ for hourly models. Hourly data gives the most accurate results, though is the most difficult to capture; monthly data can also be acceptable depending on the application, but can mask inaccuracies that can appear at hourly or daily resolutions [11].

\subsection{Calibration Outcome}

This section shall present the goodness of fit results, graphically and statistically for the calibration (before and after). The base case simulation is based on the initial values for the wall constructions ( $20 \mathrm{~mm}$ wool external, $12 \mathrm{~mm}$ wool internal) as the starting point for the calibration.

Figure 2, shows the simulated and measured data with a CV(RMSE) of 14.8\%, which is acceptable according to ASHRAE guidelines, which requires models to be under $30 \%$. Days one - four (0 to 100 hours), represent the weekdays, showing the schedul- 


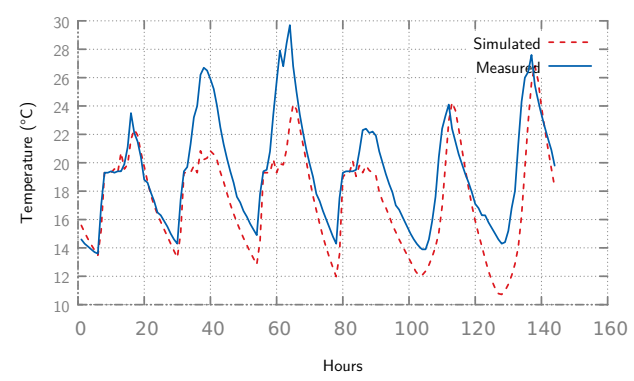

Figure 2. Temperature : Base Case : March 20th-25th 2012 : Office, Setpoint $19.3^{\circ} \mathrm{C}$ [Original Database values, $20 \mathrm{~mm}$ wool external,12mm wool internal].

ing of the heater from $6 \mathrm{~m}$ to $5 \mathrm{pm}$ to maintain a heating setpoint of $19.3^{\circ} \mathrm{C}$. The last two days represent the weekend, when the heater was off and temperature variations are due to the external climate only. Temperatures which rise above the setpoint of $19.3^{\circ} \mathrm{C}$ can be attributed to solar gains and an increase in external ambient temperature In this case, the simulator does not adequately represent this phenomenon for days two and three, though there is good agreement for day one. For this particular day, the rise in temperature is closely matched with an identical gradient for both measured and simulated data, as the heating system is actuated to reach setpoint of $19.3^{\circ} \mathrm{C}$. The setpoint is maintained until midday, when the temperature rises steeply due to external gains. The simulator represents this phenomenon matching the measured well, along with the drop in temperature. However this is not the case for subsequent days where the measured data shows temperature peaks reaching nearly $30^{\circ} \mathrm{C}$ on the third day, and gradient drops in temperature that are slightly steeper. The last two weekend days do follow the trend of the measured data, reasonably well, but the simulator again drops to a lower temperature by as much as $3^{\circ} \mathrm{C}$ by the sixth day. Figure 3 shows the measured and simulated data with a CV(RMSE) that is very high at $439 \%$, which is significantly outwith ASHRAE guidelines. The simulated values demonstrate that the model heater is having to work harder to maintain a setpoint, with repeated hourly actuations.

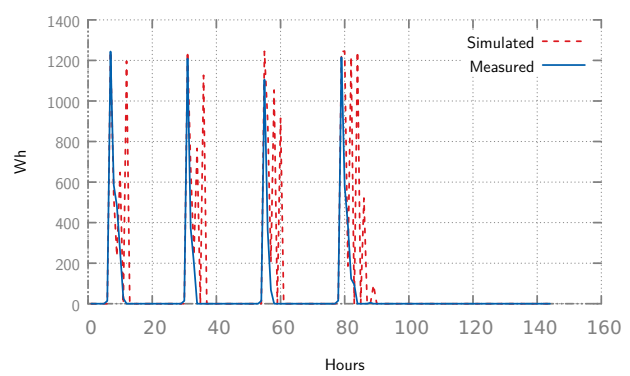

Figure 3. Heat Energy : Base Case : March 20th-25th 2012 : Office, Setpoint $19.3^{\circ} \mathrm{C}$ [Original Database values, $20 \mathrm{~mm}$ wool external, $12 \mathrm{~mm}$ wool internal]. 


\subsection{Calibrating for the Lowest CV(RMSE) for Energy Consumption}

Since the CV(RMSE) for the base case temperature response is within ASHRAE guidelines, calibration on energy consumption will be explored, in an attempt to lower it towards an acceptable level.

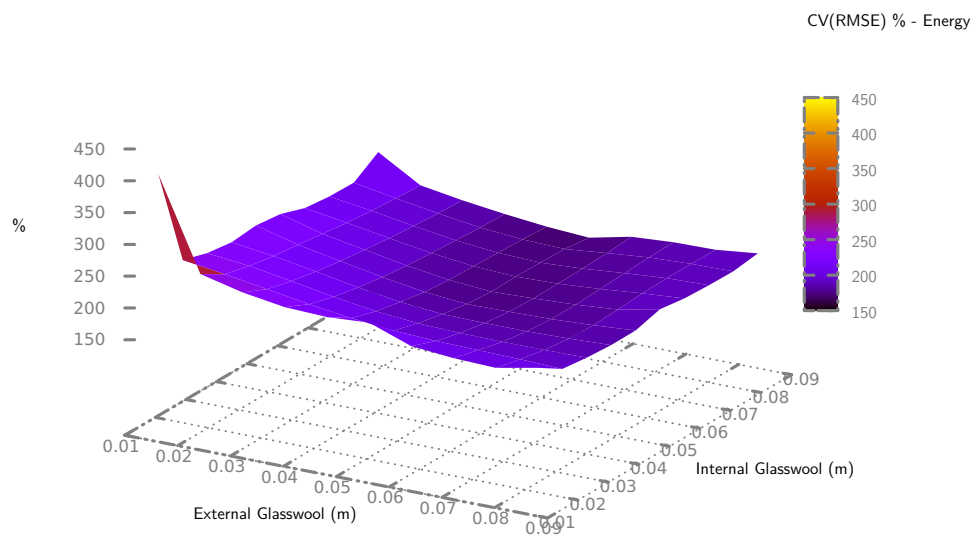

Figure 4. 3D Surface plot: Calibration Period 1 : March 20th-25th 2012: Office, Setpoint $19.3^{\circ}$ C.

The surface plot shown in Figure 4 shows the results of the calibration run for the automated calculations of CV(RMSE) for energy consumption of the Office heater and depicts the relationship between the CV(RMSE) and glasswool internal and external thickness. The highest error occurs with the lowest amounts of glasswool internal and external thickness. The plot is largely flat indicating the tuning of these parameters is overall ineffective. The results of the calibration give a CV(RMSE) for energy and temperature at $135.86 \%$ and $12.1 \%$ respectively. This revises the external insulation glasswool thickness $=59 \mathrm{~mm}$ and internal insulation thickness $=59 \mathrm{~mm}$. Figure 5 shows the measured and simulated temperature data when the model is set to these parameters. Simulated values now follow the trend of the measured data much more closely, with the $2.7 \%$ improvement in $\mathrm{CV}(\mathrm{RMSE})$ for temperature compared to the pre-calibration case. In particular, the solar gains affecting the model is more evident with the model demonstrating overheating curves that closely match the measured trends. However looking at the first day, the simulator is demonstrating higher sensitivity to overheating compared to the previous pre-calibration case. In subsequent days though the simulator represents the overheating phenomenon more closely; in particular day three reaches peak temperature to within $1^{\circ} \mathrm{C}$ (though the rise in temperature is delayed by several hours). Day four's simulated profile is almost a perfect match to the measured data, with the simulated overheating occurring at the same time, and a gradient drop in temperature that is near identical. The following two weekend days are also closely matched, though the simulator's rise in temperature is slightly delayed in comparison.

Though $135 \%$ is a high CV(RMSE) for energy response, compared to the ASHRAE guidelines, the load profile of the simulated values is consistent with the measured data, 


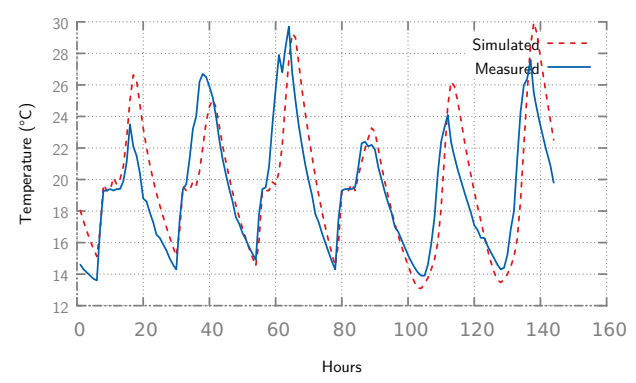

Figure 5. Temperature : Calibration Period 1 : March 20th-25th 2012 : Office, Setpoint $19.3^{\circ} \mathrm{C}$ [Calibrated Database values, $59 \mathrm{~mm}$ wool external, $59 \mathrm{~mm}$ wool internal].

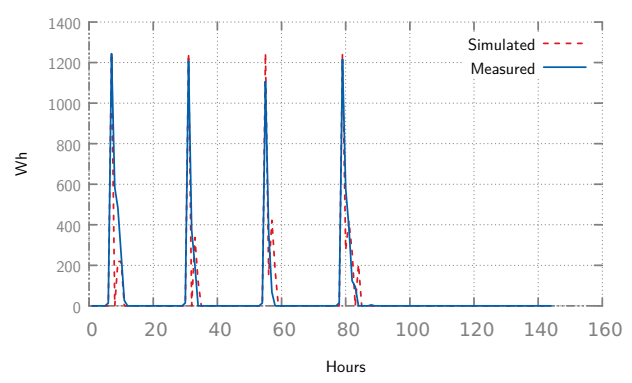

Figure 6. Heat Energy : Calibration Period 1 : March 20th-25th 2012 : Office, Setpoint $19.3^{\circ}$ C. [Calibrated Database values, $59 \mathrm{~mm}$ wool external, $59 \mathrm{~mm}$ wool internal]

as shown in Figure 6. Here the limitation of performing calibration based on CV(RMSE) as per guidelines, at the hourly level for electrical heating loads is evident. However the CV(RMSE) for temperature response yields a low $12.1 \%$ for CV(RMSE), which is well within the guidelines. Following a potentially well matched initial load profile as seen in Figure 6 and Figure 5, the heating works the hardest first thing each morning to reach setpoint, but later actuations can significantly vary between time periods. Clearly this due to the fact $\mathrm{CV}$ (RMSE) compares predicted with measured data point to point, which may be appropriate for hourly temperatures but not highly variable energy delivery. [15] also recognised that evaluating calibration accuracy at small time scales (or scales where conditions are very variable) using CV(RMSE) is not appropriate. Graphically and statistically, the simulated temperature profile of this room could suggest this model is calibrated. In previous calibration studies (such as those carried out by Tahmasebi et al.) who only considered a subset of the building and an averaged zone temperature profile for a single floor of a building, a match can indeed be attained to the measured data, however may not be the case when taking a wider view across the whole building - the effects of ceiling and floor dynamics must also be considered. Furthermore, graphical analysis is equally important, particularly for temperature response, since a small change in $\mathrm{CV}(\mathrm{RMSE})$ can actually lead to a significantly better fit to the measured data when shown graphically against the simulator.

The importance of considering whole house dynamics is highlighted by looking at the adjacent Living Room. Figure 7 shows the hourly measured v simulated temperature 
data with the chosen calibrated values, and demonstrates how the uncertain model is failing at predicting the temperature response for this zone.

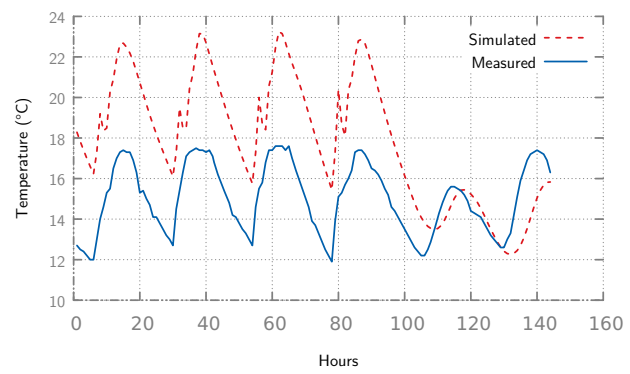

Figure 7. Temperature : March 20th-25th 2012 : Living Room, Setpoint $17.3^{\circ} \mathrm{C}$ [Calibrated Database values, $59 \mathrm{~mm}$ wool external, $59 \mathrm{~mm}$ wool internal].

Figure 7 shows a large disparity between the temperatures of the measured and simulated values by as much as $6^{\circ} \mathrm{C}$. The simulator is consistently overheating the zone, suggesting that there may issues with heat transfer, as it appears the heat is not escaping to allow the temperature to equalise to setpoint.

Furthermore, in terms of energy response shown in Figure 8, the measured values indicate that the heating remains on for the duration of the day, thus showing the heater having to work harder to maintain setpoint. In contrast though, the simulator heat load is minor in comparison, though the simulator temperature response indicates significant overheating, further demonstrating how ineffective the uncertain model is, since it is using a fraction of energy compared to what was measured. As for modelling uncertainty, airflow has not been considered. This could lead a modeller to apply ESP-r's standard scheduled airflow technique in an attempt to 'force' heat transfer. An example of this is shown in Figure 9 with the application of a scheduled airflow rate of 2.5 Air Change Rate $(\mathrm{ACH})$. This results in the simulator temperature response being more erratic, though the differences between peak temperatures between the simulated and measured data has reduced. There could be a temptation to further manipulate the $\mathrm{ACH}$ rate in the absence of an air flow model, but this would certainly lead to a model far removed from reality.

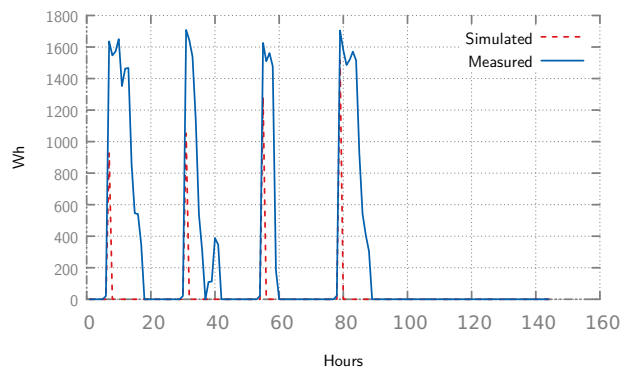

Figure 8. Heat Energy : March 20th-25th 2012. Living Room, Setpoint $17.3^{\circ} \mathrm{C}$ [Calibrated Database values, $59 \mathrm{~mm}$ wool external, $59 \mathrm{~mm}$ wool internal]. 


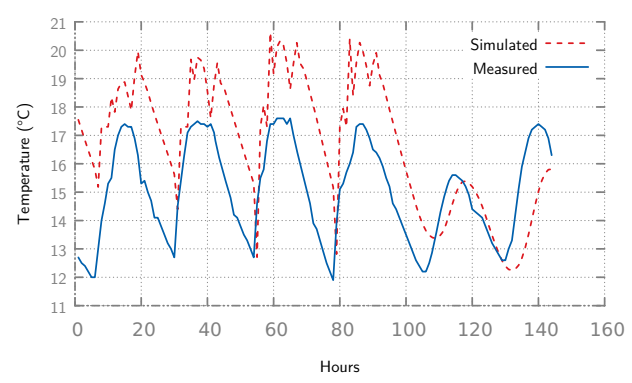

Figure 9. Temperature : March 20th-25th 2012 : Living Room, Setpoint $17.3^{\circ} \mathrm{C}$ [Calibrated Database values, $59 \mathrm{~mm}$ wool external, $59 \mathrm{~mm}$ wool internal] 2.5 airchanges/hour.

\section{Conclusion}

Due to the complexity of interactions in a building model, calibration should only be used to determine a few uncertain parameters. In particular when applying ASHRAE guidelines for temperature fit, a model may appear calibrated when looking at the temperature response of an individual zone, but may not be the case upon deeper investigation of other zones. The uncertain model makes some assumptions on the structure of the house. The most prevalent assumption, is that there is no roof zone, which has been approximated as an external boundary. There is uncertainty in the choice of unknown parameters (insulation thickness), which can lead to a fit to the data, even if they are out of the actual range (maximum end of range chosen to be $90 \mathrm{~mm}$, whereas the actual was $100 \mathrm{~mm}$ ). Finally in terms of modelling uncertainty, a simplified approach to introducing airflow has been applied to investigate if the characteristics can be improved, which can be seen as attempt at 'fudging', and not conducive to produce a reasonable solution. The problem with this model is most apparent when observing the large disparity in temperature and energy response contained in the the Living Room results, and demonstrates some of pitfalls when relying on calibration to try and achieve goodness of fit to tune the model. A satisfying solution may be achieved for one zone in the model, as can be seen with the high goodness of fit with the temperature response in the Office zone, but on closer inspection, may not be the case in other zones, as shown in the Living Room zone results. Furthermore, the difficulty with calibrating on energy use when using electrical heating power has been demonstrated, and that $\mathrm{CV}$ (RMSE) may not be the most ideal metric to ascertain 'goodness of fit', when doing hourly comparisons of heat delivery. Though it may be possible to further adjust parameters on the model to achieve a better fit, and may not necessarily represent reality, and therefore may not be able to predict adequately when presented with other data sets and use cases.

\section{References}

[1] Strachan PA, Kokogiannakis G, Macdonald IA. History and development of validation with the ESPr simulation program. Building and Environment. 2008 Apr;43(4):601-609. Available from: http: //www.sciencedirect.com/science/article/pii/S0360132306003313.

[2] Bengea SC, Kelman AD, Borrelli F, Taylor R. Model Predictive Control for Mid-Size Commercial Building HVAC : Implementation, Results and Energy Savings. HVAC\&R Research. 2014;20(1). 
[3] Lehmann B, Gyalistras D, Gwerder M, Wirth K, Carl S. Intermediate complexity model for Model Predictive Control of Integrated Room Automation. Energy and Buildings. 2013 Mar;58:250-262. Available from: http://www.sciencedirect.com/science/article/pii/S0378778812006561.

[4] Široký J, Oldewurtel F, Cigler J, Prívara S. Experimental analysis of model predictive control for an energy efficient building heating system. Applied Energy. 2011 Sep;88(9):3079-3087. Available from: http://www.sciencedirect.com/science/article/pii/S0306261911001668.

[5] Clarke J. Energy Simulation in Building Design. Routledge; 2001. Available from: http://www.elsevier.com/books/energy-simulation-in-building-design/clarke/ 978-0-7506-5082-3.

[6] Li S, Song Z, Zhou M, Lu Y. Sensor data quality assessment for building simulation model calibration based on automatic differentiation. In: 2013 IEEE International Conference on Automation Science and Engineering (CASE). IEEE; 2013. p. 752-757. Available from: http: //ieeexplore. ieee.org/ articleDetails . jsp?arnumber $=6654061$.

[7] Robertson J, Polly B, Collis J. Evaluation of Automated Model Calibration Techniques for Residential Building Energy Simulation Evaluation of Automated Model Calibration Techniques for Residential Building Energy Simulation. NREL; 2013. September.

[8] Reddy TA, Maor I, Panjapornpon C. Calibrating Detailed Building Energy Simulation Programs with Measured DataPart I: General Methodology (RP-1051). HVAC\&R Research. 2007 Mar;13(2):221-241. Available from: http://www.tandfonline.com/doi/abs/10.1080/10789669.2007.10390952.

[9] Reddy A, Maor I. ASHRAE Research Project 1051- RP Procedures for Reconciling ComputerCalculated Results With Measured Energy Data; 2006. iii.

[10] ASHRAE. Measurement of Energy and Demand Savings. ASHRAE; 2002.

[11] Raftery P, Keane M, Costa A. Calibrating whole building energy models: Detailed case study using hourly measured data. Energy and Buildings. 2011 Dec;43(12):3666-3679. Available from: http: //www.sciencedirect.com/science/article/pii/S0378778811004415.

[12] Tahmasebi F, Zach R, SchußM, Mahdavi A. Simulation Model Calibration : An Optimization-based Approach. In: International Building Performance Simulation Association; 2012. p. 386-391.

[13] Seeam A, Laurenson D, Usmani A. Evaluating the potential of simulation assisted energy management systems: A case for electrical heating optimisation. Energy and Buildings. 2018;174:579-586.

[14] de Wit S, Augenbroe G. Analysis of uncertainty in building design evaluations and its implications. Energy and Buildings. 2002 Oct;34(9):951-958. Available from: http://www. sciencedirect . com/ science/article/pii/S0378778802000701.

[15] Ruiz Flores R, Lemort V. Calibration of Building Simulation Models: Assessment of Current Acceptance Criteria. 2014 Sep;Available from: http://orbi .ulg.ac.be/handle/2268/168878. 